\title{
\#shareEGU20
}

$$
\text { 4- } 8 \text { May } 2020
$$

\section{Refactoring the memory access pattern to improve computational performance in NEMO}

I.Epicoco, F. Mele, S. Mocavero, M. Chiarelli, A. D'Anca, G. Aloisio

OS4.8 - Numerical modelling of the ocean: new scientific advances in ocean models to foster exchanges within NEMO community and contribute to future developments 


\section{NEMO Ocean Model}

Single core performance of the NEMO model is limited by memory access and poor exploitation of vector processing units on modern HPC architectures

The analysis of the memory access pattern shows that many repeated accesses occur for reading values not available in cache -> high rate of cache miss!!!

G(2) $4 L$ Goal of the work: enhance the exploitation of the cache memory of the modern parallel architectures through the loop fusion approach 


\section{Single core performance - Loop fusion}

- Loop fusion technique aims at better exploiting the cache memory by fusing DO loops together

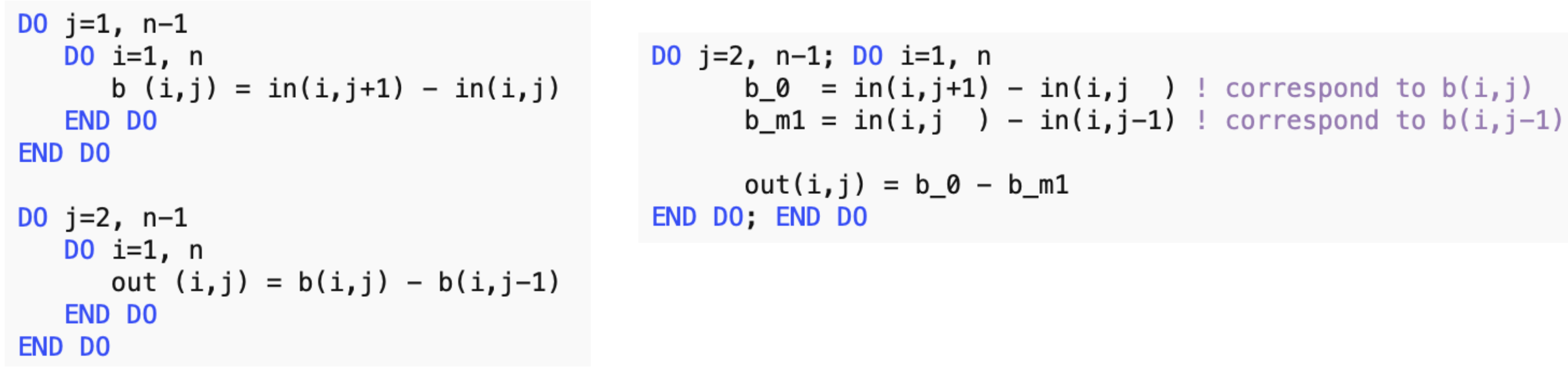

- advantages: reduction of cache misses and reduction of the memory footprint

- disadvantage: due to data dependencies redundant operations are needed 


\section{Single core performance - Loop fusion}

- Loop fusion approach has been applied on the NEMO MUSCL advection kernel

- Three different levels of fusion have been implemented

Dprototype1: has the maximum level of fusion with redundant operations

Dprototype2: introduces the buffers rotation ${ }^{1}$ in the outer loop

$\square$ prototype3: uses the buffers rotation in the outer and middle loop

DO $j=1, n-1$

DO $\begin{aligned} & i=1, n \\ & b(i, j)=f b(i, j+1)-f b(i, j)\end{aligned}$

END DO
END DO
DO $j=2$,

$j=2, n$
$D O i=1$,

$s(i, j)=b(i, j)+b(i, j-1)$

END DO

DO $\mathrm{j}=2, \mathrm{n}-1$

$j=2, n-1$
$D O i=1, n$

$f a(i, j)=s(i, j)-s(i, j-1)$ b_m2 $(:)=f b(:, 2)-f b(:, 1)$

b $m 1(:)=f b(:, 3)-f b(:, 2)$

s_m1 $(:)=$ b_m1 (:) + b_m2(: $)$

DO $j=3, n-1$

$b(:)=f b(:, j+1)-f b(:, j)$

$s(:)=b(:)+b \_m 1(:)$

DO $i=1, n$

tmp => b m2; b m2 $\Rightarrow$ b_m1; b m1 $\Rightarrow b ; b=t m p$

tmp => s_m1; s_m1 $=>$ s; s $=>$ tmp END DO
${ }^{1}$ buffers rotation technique avoid redundant operations by adopting pointers to arrays and implementing a rotation at each loop iteration as shown in the figure 


\section{First approach - prototype1}

- The halo exchange is moved before all DO-loops (at the beginning of the routine)

- The halo region needs to be extended up to two halo lines

- The advective trend is computed for each single (ji, jj, jk, jn) grid cell within a single big DO-loop

- This approach implies also a duplication of the calculus up to a factor 3 


\section{First approach - prototype1}
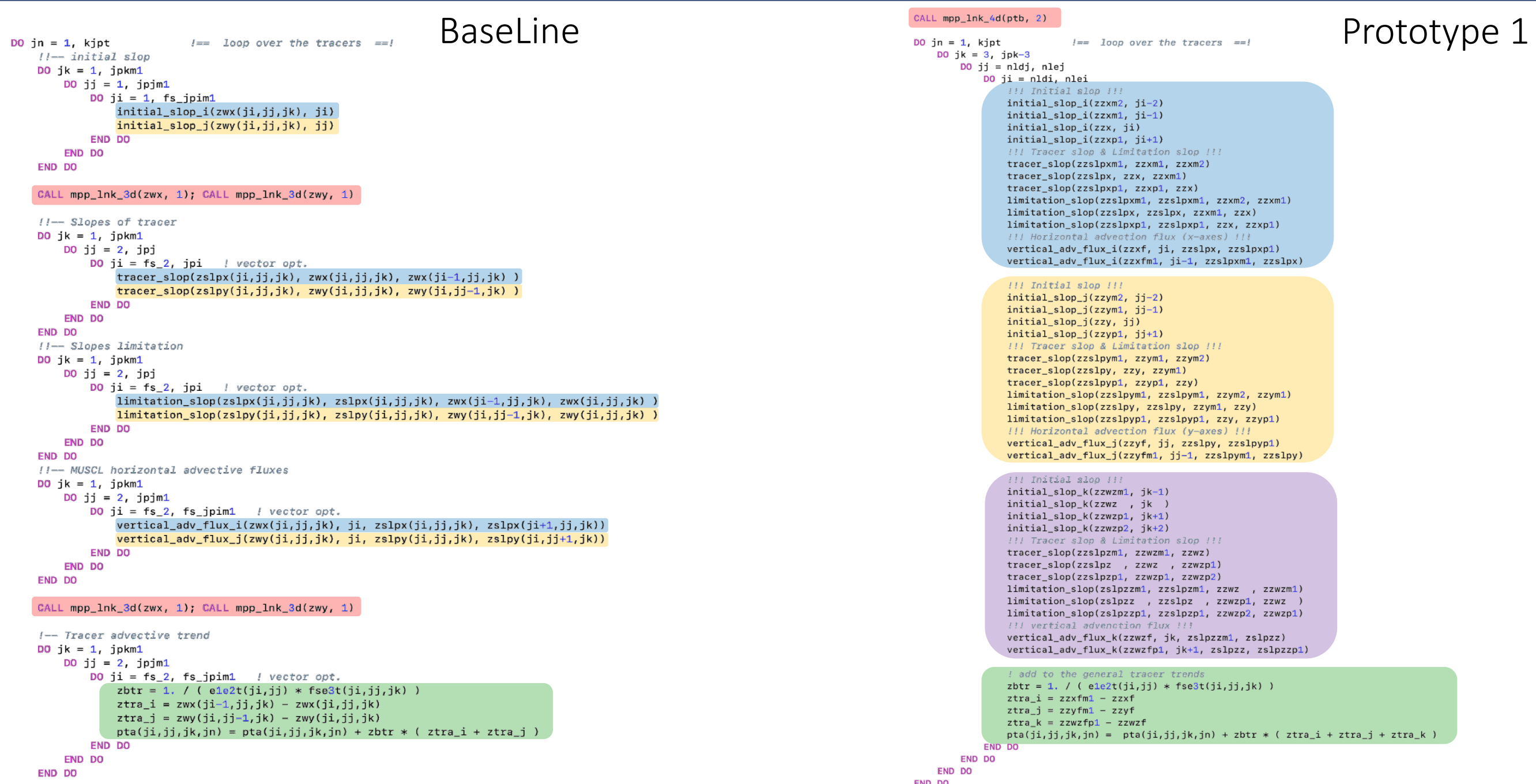


\section{Second approach - prototype2}

- Along the vertical direction:

- The advective flux at level $\mathrm{jk}=1$ is computed before the loop over $\mathrm{jk}$ (we call this flux $\mathrm{F}_{\mathrm{jk}-\mathrm{1}}$ )

- Inside the jk loop, the flux at level jk (which we call $F_{j k}$ ) is computed

- We use $F_{j k-1}$ and $F_{j k}$ to calculate the advective trend at level $j k$ and to update the RHS variable at level $\mathrm{jk}$

- Before incrementing the jk level, we update the flux at level jk-1: $F_{j k}->F_{j k-1}$

- This approach reduces the number of redundant operations, but it introduces a data dependencies in the jk loop, hence the jk loop can not be vectorized, neither executed in parallel. 


\section{Second approach - prototype2}
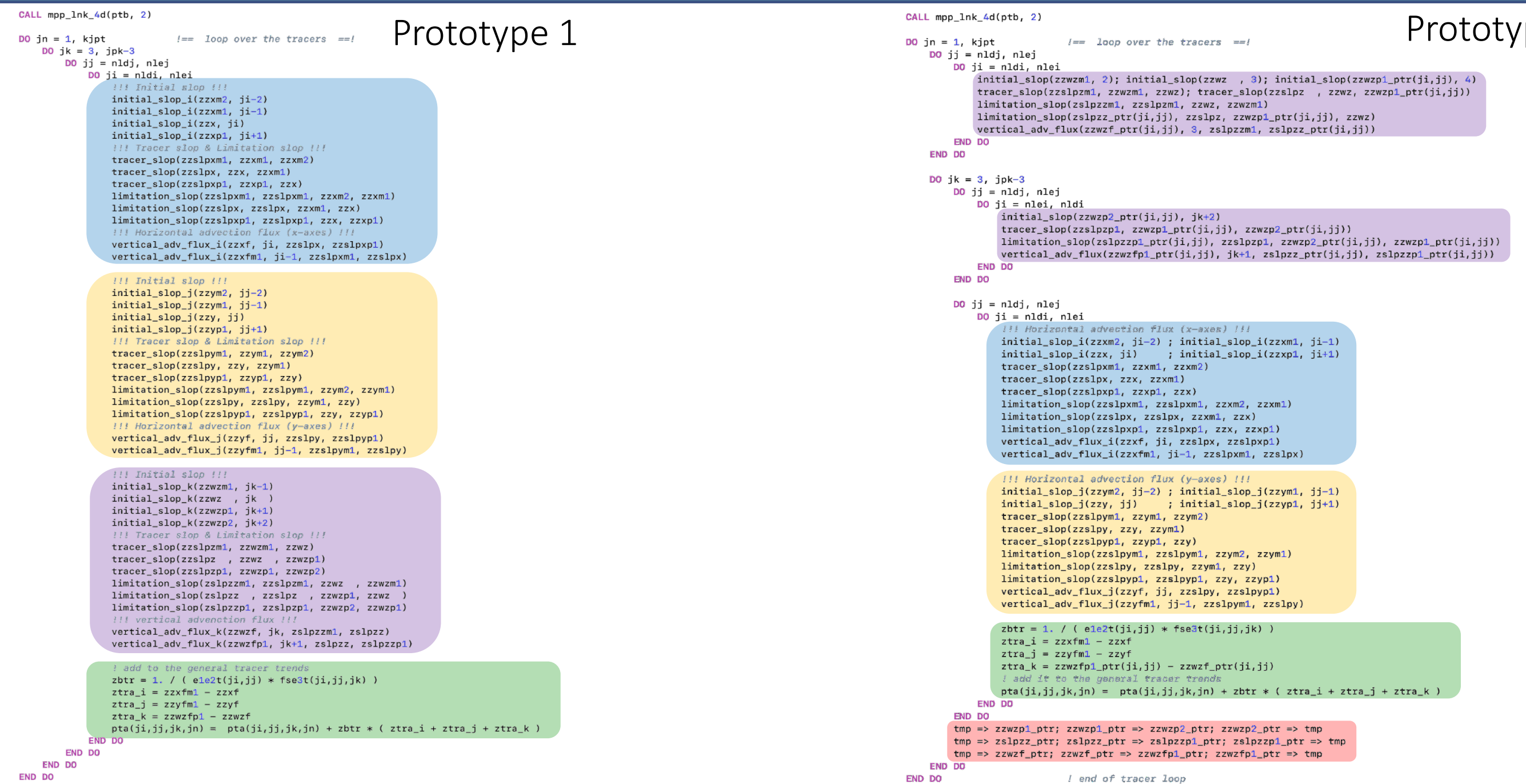

\# shareEGU20 - Refactoring the memory access pattern to improve computational performance in NEMO 


\section{Third approach - prototype3}

- Along the horizontal direction:

- In the jk loop, before updating the RHS variable with the advective trend, the fluxes for the whole horizontal domain are computed

- The fluxes are hence used to compute the advective trend and to update the RHS variable

- This approach further reduces the number of redundant operations. 


\section{Third approach - prototype3}

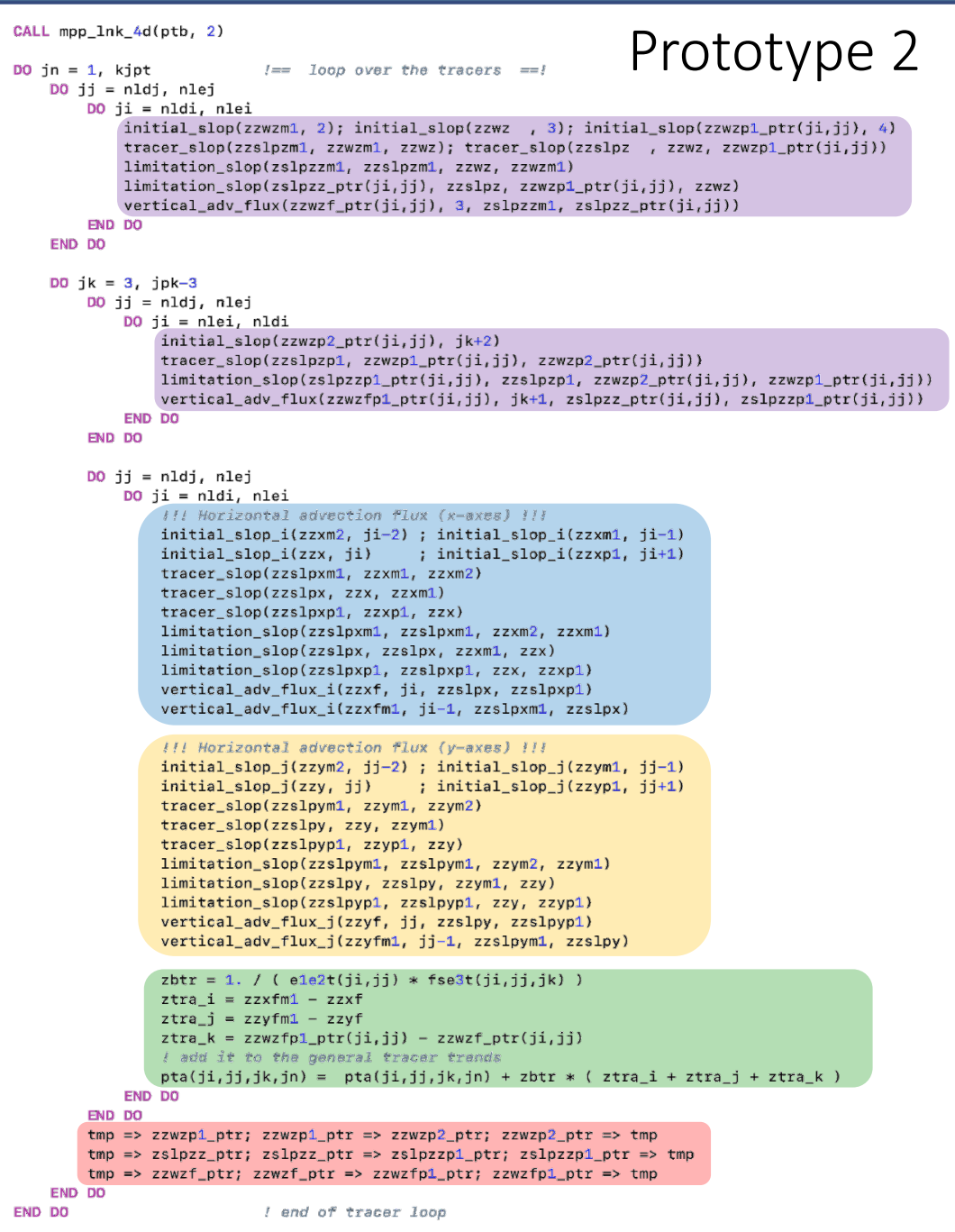

\# shareEGU20 - Refactoring the memory access pattern to improve computational performance in NEMO 


\section{Preliminary Performance Analysis}

- Global domain: $2240 \times 1500 \times 31$ points

- Smallest sub-domain (with 1024 cores): 74 x 51 x 31 points

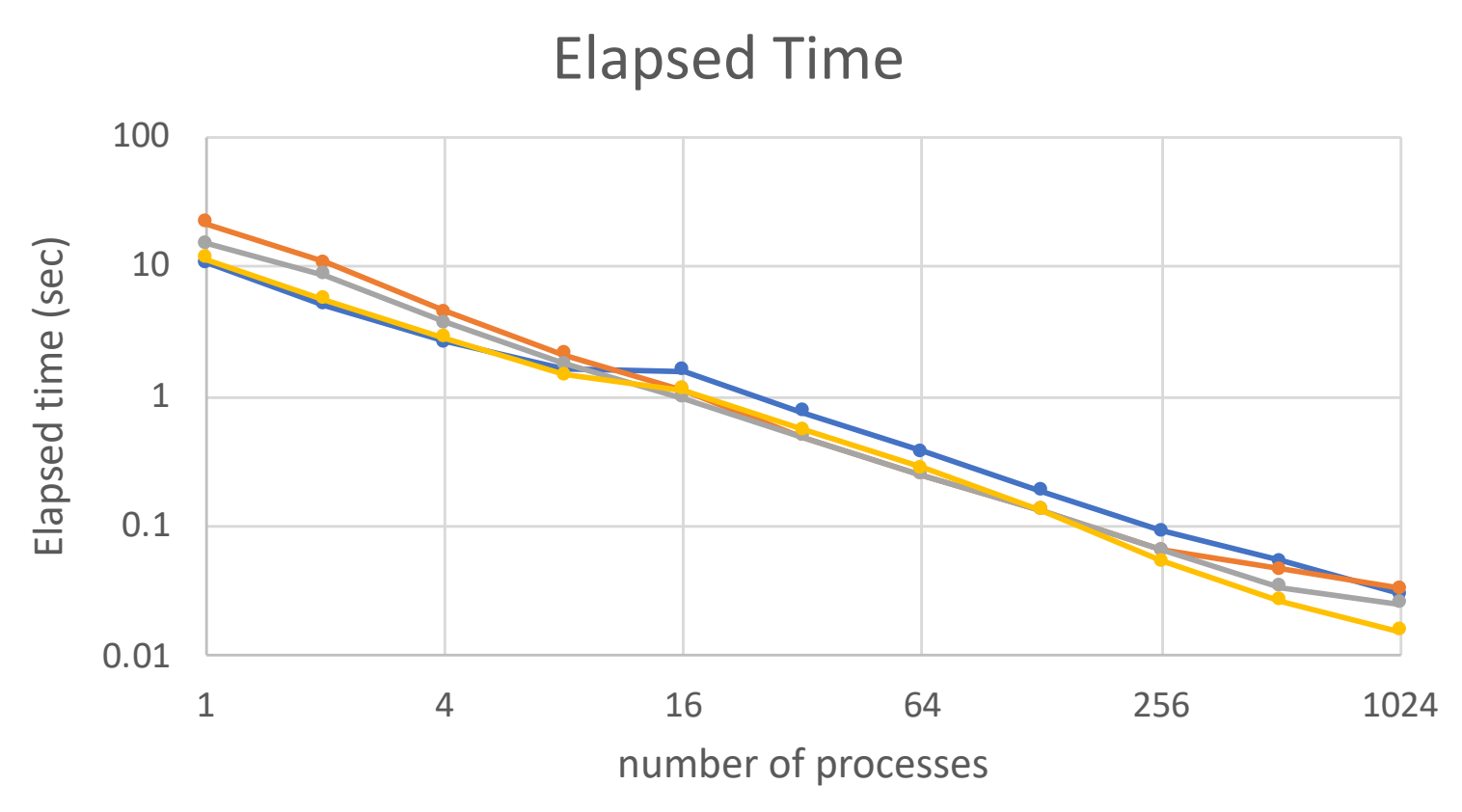

$\rightarrow$ Base $\rightarrow$ Loop_Fusion Proto1 $\rightarrow$ Loop_Fusion Proto $2 \multimap$ Loop_Fusion Proto3

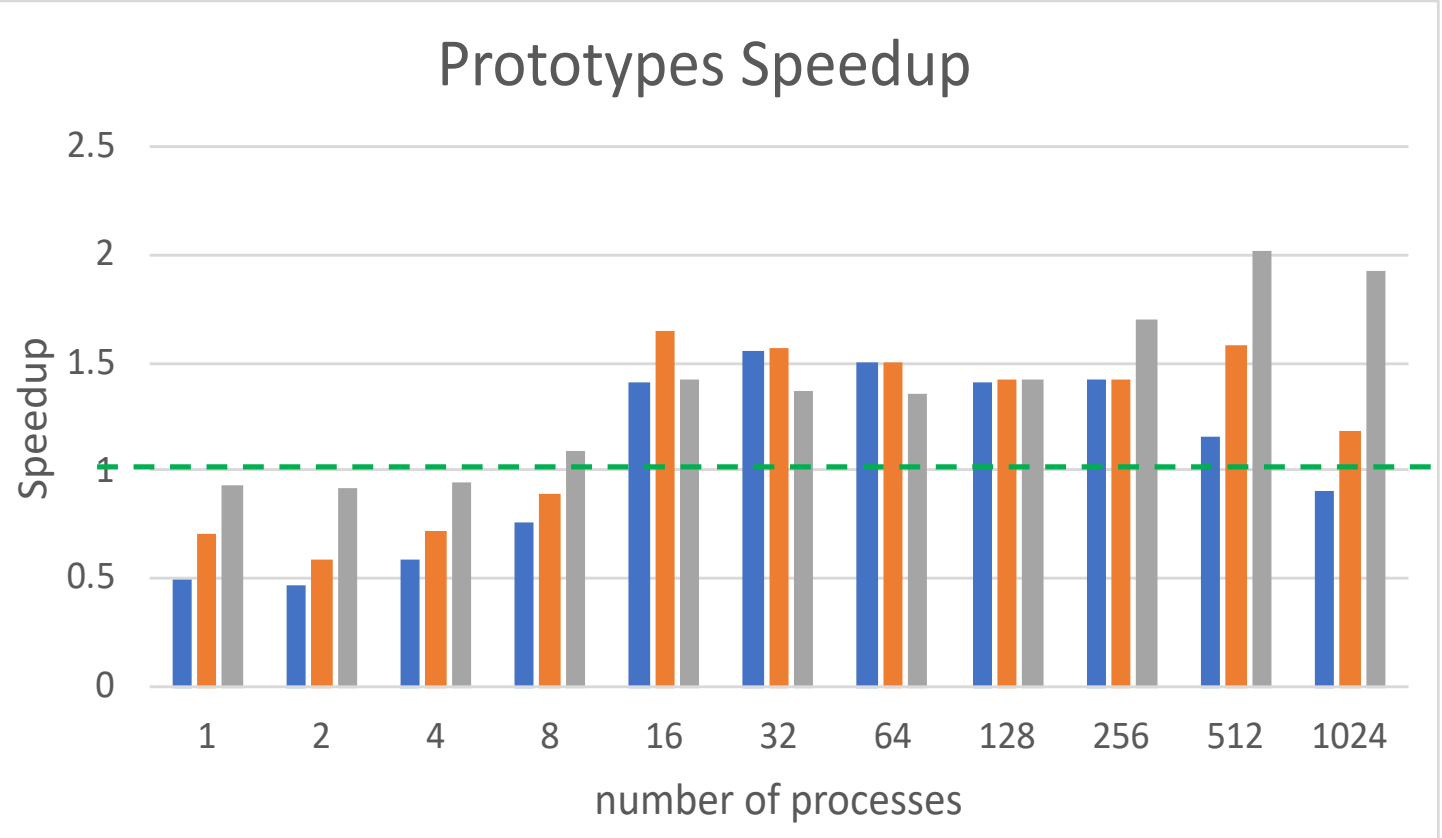

- Loop_Fusion Proto1 _Loop_Fusion Proto2 


\section{Preliminary Performance Analysis}

- Global domain: $2240 \times 1500 \times 31$ points

- Smallest sub-domain (with 1024 cores): $74 \times 51 \times 31$ points
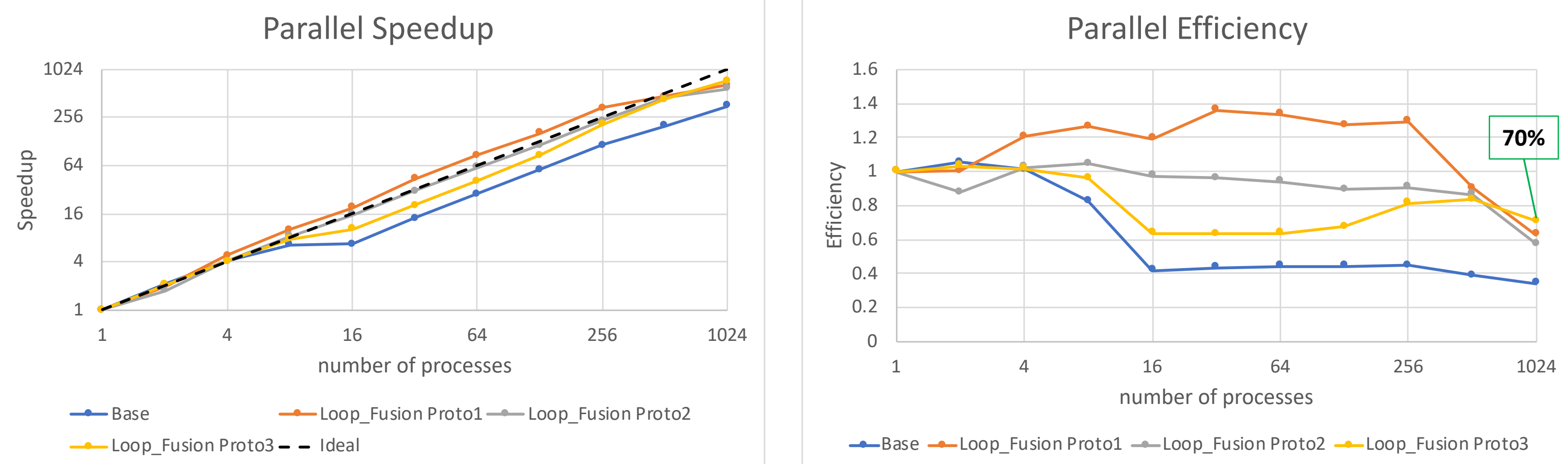

\# shareEGU20 - Refactoring the memory access pattern to improve computational performance in NEMO 


\section{Preliminary Performance Analysis}

- Global domain: $2240 \times 1500 \times 31$ points

- Smallest sub-domain (with 1024 cores): $74 \times 51 \times 31$ points

L3 Total Cache Misses

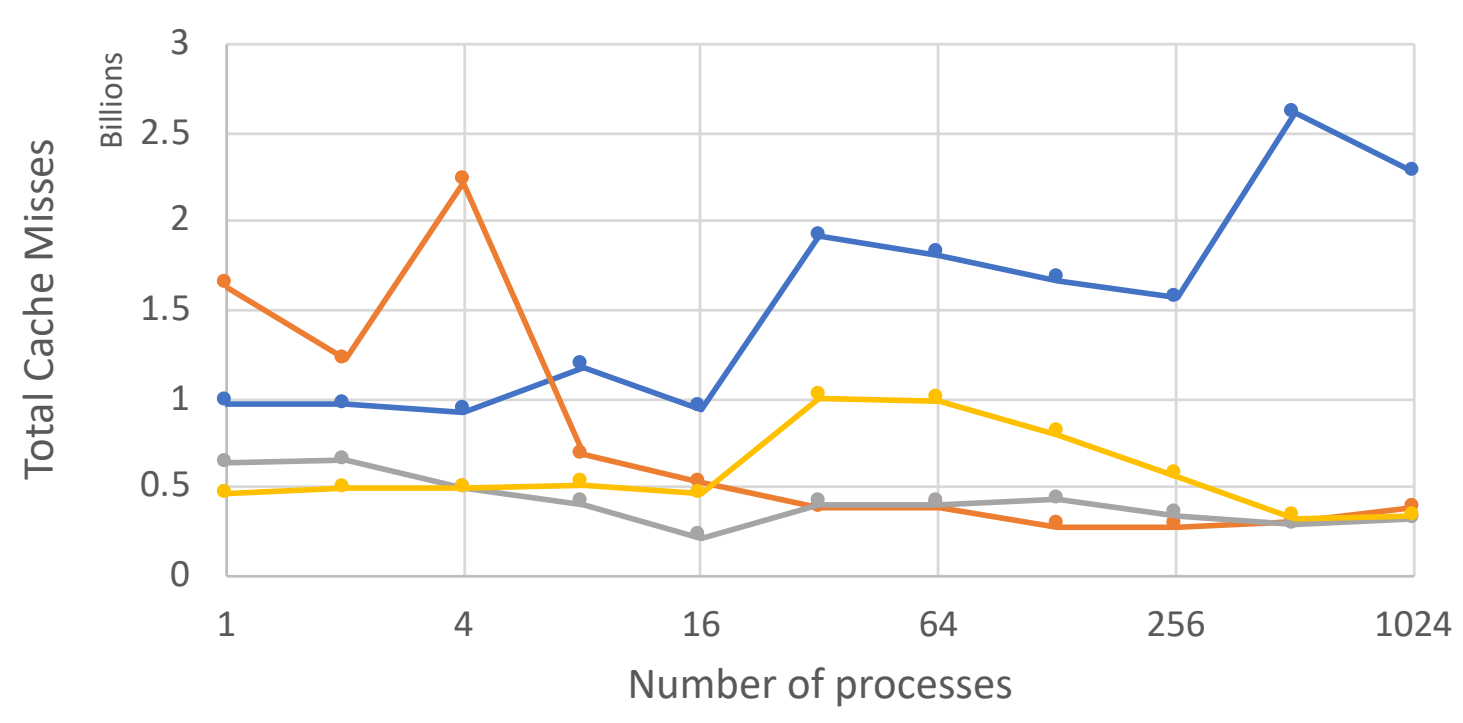

-Base $\_$Loop Fusion Proto1 $\_$Loop Fusion Proto2 - Loop Fusion Proto3

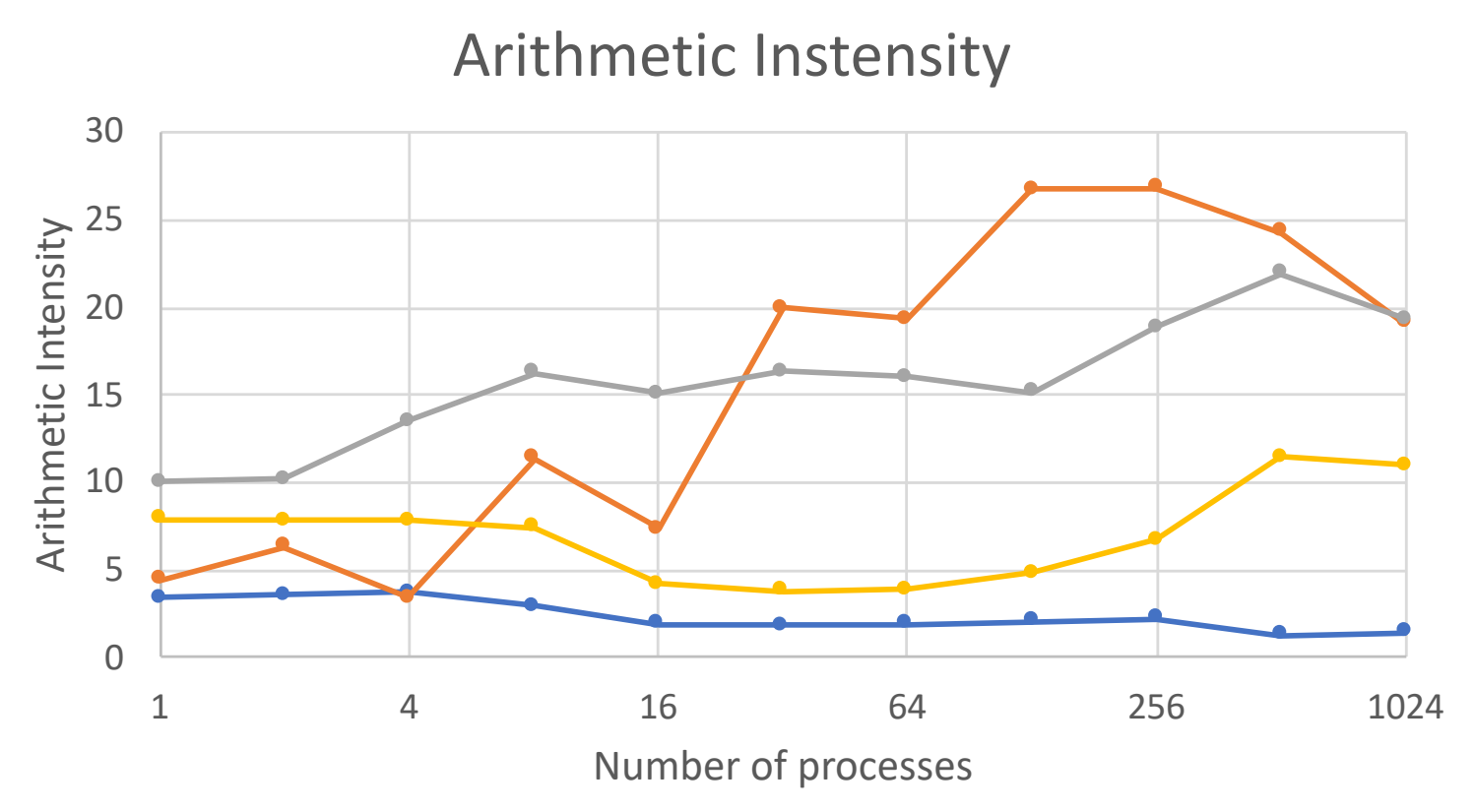

$\rightarrow$ Base $\rightarrow$ Loop Fusion Proto1 $\rightarrow$ Loop Fusion Proto $2 \rightarrow$ Loop Fusion Proto3 


\section{Preliminary Performance Analysis}

- Global domain: $70 \times 46 \times 19$ points

- Sub-domain with 64 cores: $13 \times 10 \times 19$ points

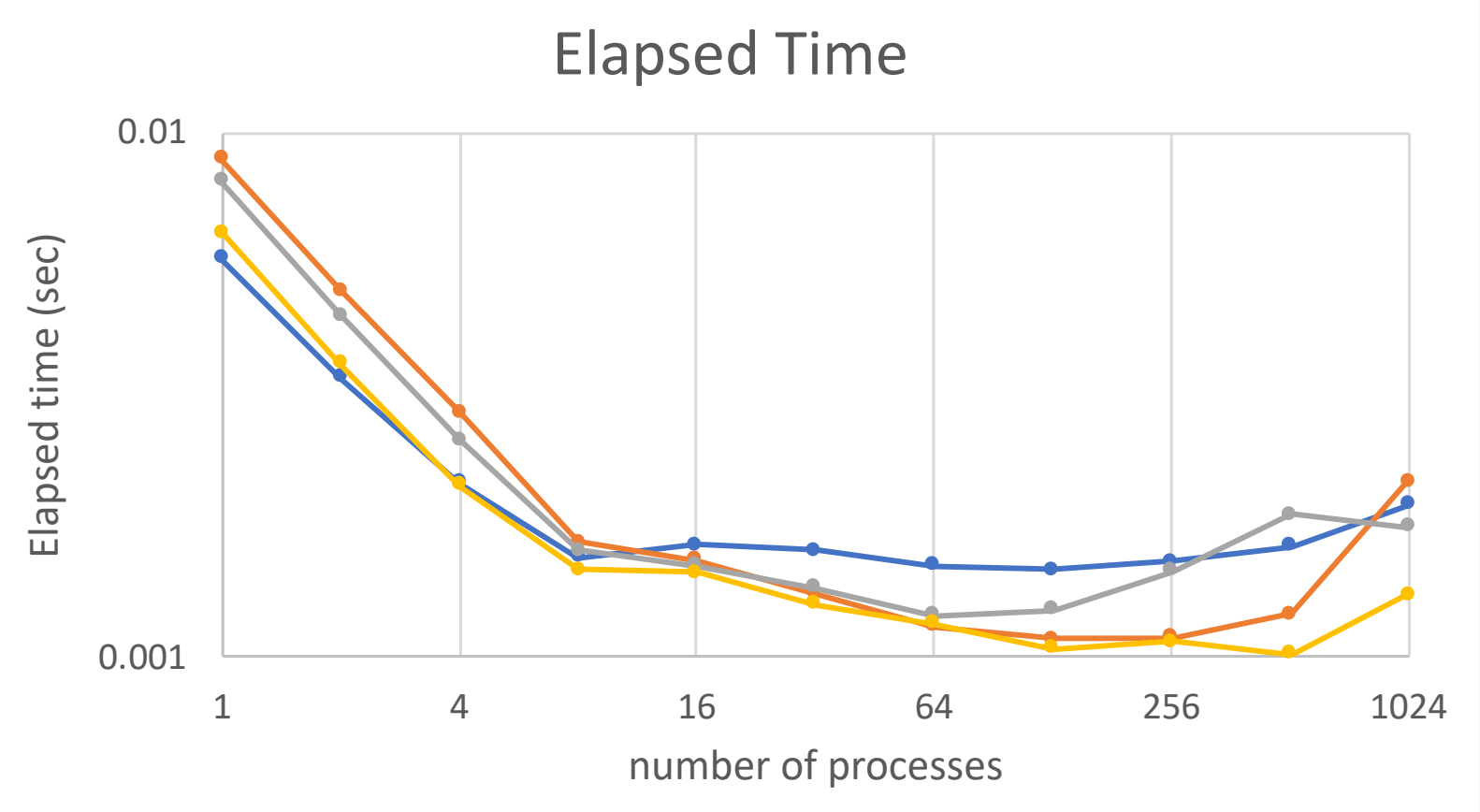

$\multimap$ Base $\rightarrow$ Loop_Fusion Proto $1 \multimap$ Loop_Fusion Proto $2 \multimap$ Loop_Fusion Proto 3

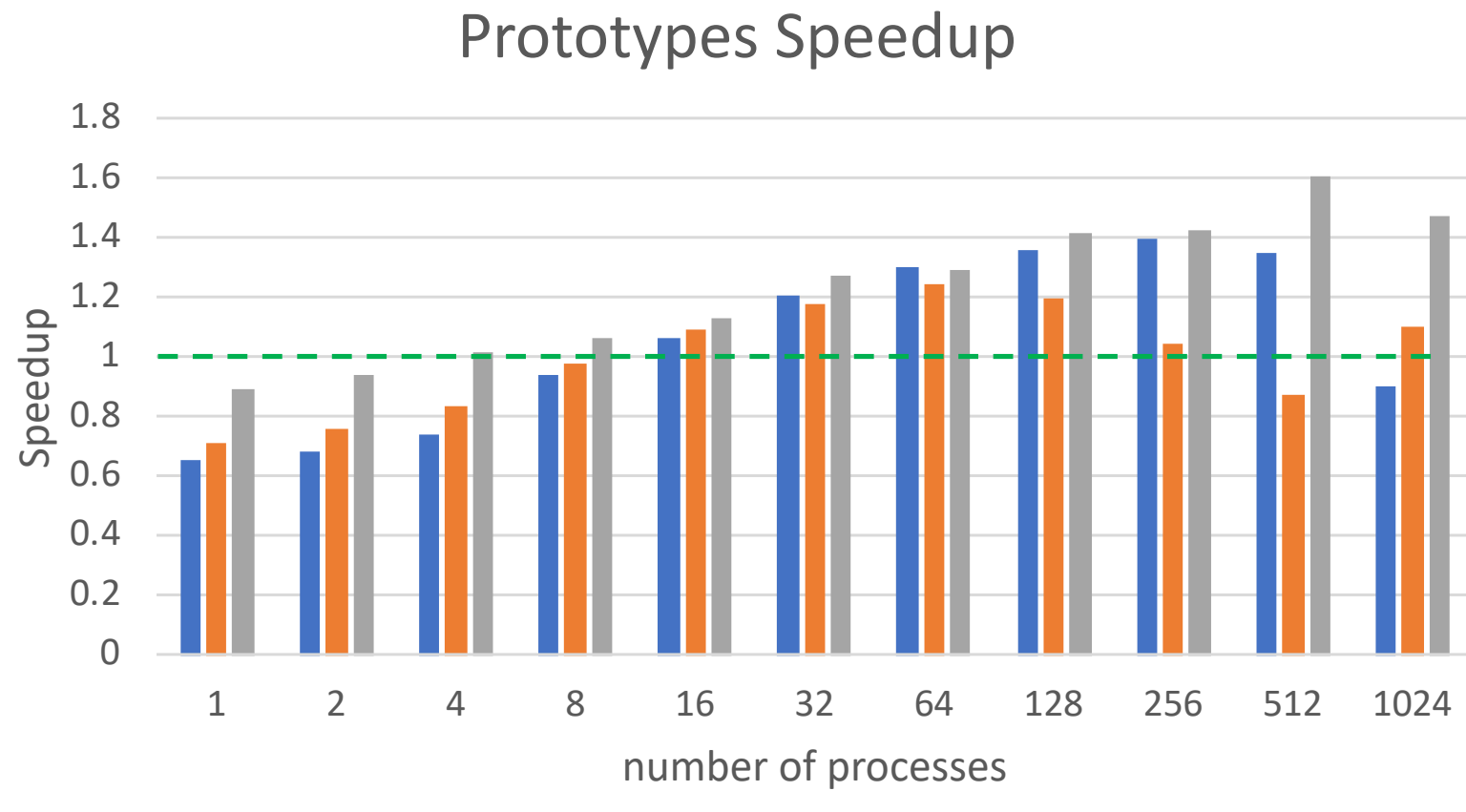

- Loop_Fusion Proto1 _ Loop_Fusion Proto2 


\section{Preliminary Performance Analysis}

- Global domain: $70 \times 46 \times 19$ points

- Sub-domain with 64 cores: 13 x $10 \times 19$ points
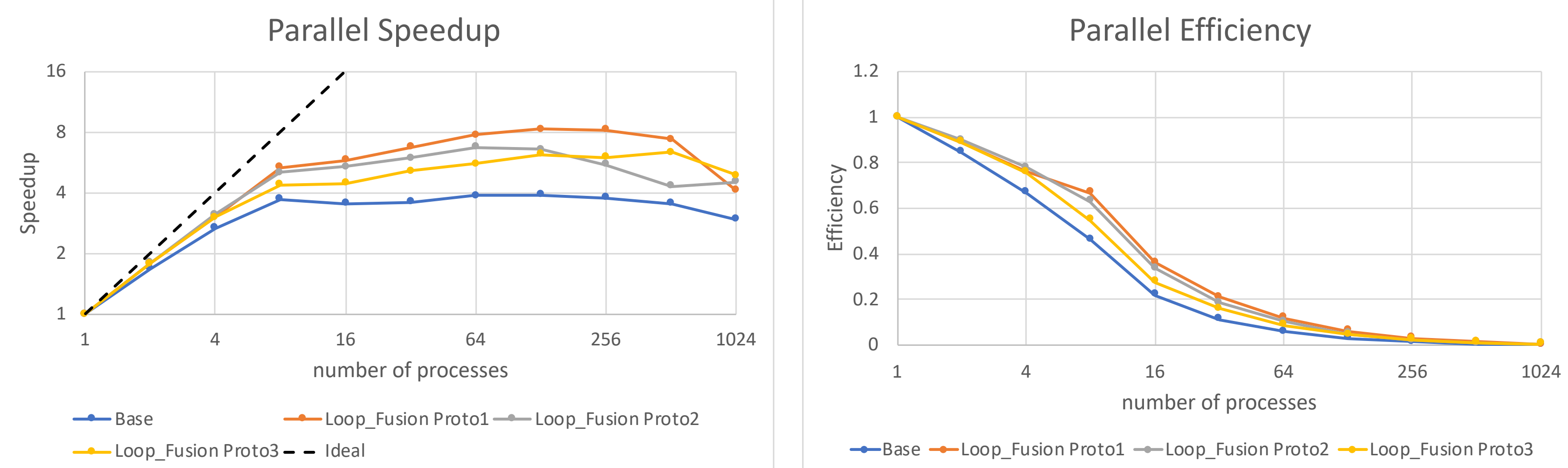

$\multimap$ Base $\rightarrow$ Loop_Fusion Proto $1 \multimap$ Loop_Fusion Proto $2 \multimap$ Loop_Fusion Proto 3

\# shareEGU20 - Refactoring the memory access pattern to improve computational performance in NEMO 


\section{Preliminary Performance Analysis}

- Prototypes 1 and 2 provide a good improvement up to 256 cores then the redundant operations lead to a loss of performance

- Prototypes 3 improves parallel efficiency by $\sim 30 \%$ on 1024 cores

- This approach enhanced the vectorization level and the cache reuse, reducing L3 Total Cache Misses by $80 \%$ on 1024 cores

- Loop-fusion is strictly linked to the computing architecture $\rightarrow$ A fully portable performance improvement can be ensured by the adoption of a DSL. 


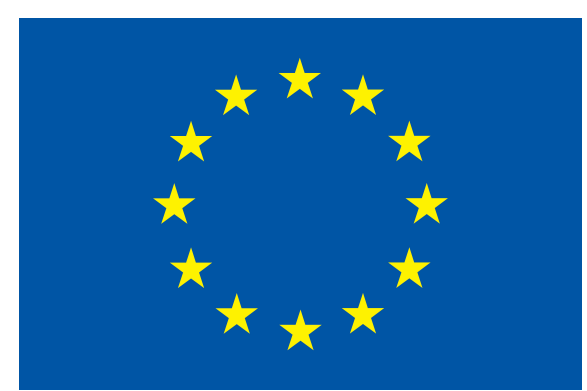

The IS-ENES3 project has received funding from the European Union's Horizon 2020 research and innovation programme under grant agreement $N^{\circ} 824084$

\section{https://is.enes.org}

